\title{
La enseñanza por pares, una estrategia didáctica para el aprendizaje de la electrocardiografía básica
}

\section{Peers teaching a didactic strategy for the learning of basic electrocardiography}

\author{
Juan Carlos Méndez-Velásquez, Jaime Pérez-Giraldo • Medellín (Colombia)
}

DOI: https://doi.org/10.36104/amc.2021.1971

\section{Resumen}

La enseñanza de la electrocardiografía es un área de gran importancia en la formación de los estudiantes de pregrado, para los docentes es una preocupación constante que los médicos en formación adquieran las competencias para la lectura e interpretación del electrocardiograma (ECG). El presente estudio describe e indaga sobre cómo la estrategia didáctica de la enseñanza por pares mejora los procesos de aprendizaje de los estudiantes de segundo año de formación médica. Se halló evidencia de que la enseñanza por pares favorece el aprendizaje significativo de la fisiología cardiaca y del electrocardiograma, en particular, porque permite la adquisición de habilidades y destrezas a los estudiantes en la lectura del ECG y mejora la comprensión e interpretación de dicho examen; además, propicia relaciones horizontales entre pares que facilitan el desarrollo de la autonomía, el autoaprendizaje y el trabajo en equipo. (Acta Med Colomb 2021; 46. DOI: https:// doi.org/10.36104/amc.2021.1971).

Palabras clave: educación médica, enseñanza por pares, métodos de enseñanza, aprendizaje significativo, electrocardiografía.

\section{Summary}

Teaching electrocardiography is a very important area in undergraduate student training. A constant concern of professors is for physicians in training to acquire competence in reading and interpreting electrocardiograms (EKGs). This study describes and explores how the didactic strategy of peer teaching improves learning processes for second-year medical students. Evidence was found that peer teaching fosters significant learning of cardiac physiology and electrocardiograms, in particular, because it allows students to acquire abilities and skills in EKG reading, and improves the understanding and interpretation of this test. In addition, it promotes horizontal peer relationships which facilitate the development of autonomy, self-learning and teamwork. (Acta Med Colomb 2021; 46. DOI: https://doi.org/10.36104/amc.2021.1971).

Key words: medical education, peer teaching, teaching methods, significant learning, electocardiography.
Dr. Juan Carlos Méndez-Velásquez: Bacteriólogo y Laboratorista Clínico, MsC Ciencias Básicas Biomédicas, MsC Educación Superior en Salud. Profesor Departamento de Fisiología y Bioquímica; Dr. Jaime Pérez.-Giraldo: Medico. Especialidad en Medicina Deportiva, MsC Educación Superior en Salud, Jefe del Departamento de Fisiología y Bioquímica.

Facultad de Medicina, Universidad de Antioquia. Grupo de Investigación EDUSALUD. Medellín (Colombia).

Correspondencia: Dr. Juan Carlos MéndezVelásquez.

E-Mail: juan.mendez@udea.edu.co

Recibido: 27/VII/2020 Aceptado: 18/XI/2020

\section{Introducción}

Los currículos de medicina tienen entre sus objetivos de formación el desarrollo de habilidades y destrezas cognitivas, actitudinales y bioéticas, las cuales se ven reflejadas en una acertada comprensión del cuadro clínico de los pacientes y su correlación con las ayudas diagnósticas (1).

El electrocardiograma (ECG) es una ayuda diagnóstica de gran valor para la detección de patologías cardiacas, como la enfermedad isquémica del corazón, que según estudios epidemiológicos es la primera causa de muerte en Colombia y el mundo (2). En el ámbito educativo es objeto de investigación encontrar las estrategias didácticas más adecuadas para la enseñanza de la electrocardiografía, su interpretación y comprensión de los resultados. Algunos estudios han demostrado cierta variabilidad en el aprendizaje del ECG y recomiendan utilizar estrategias de enseñanza 
como talleres, cursos virtuales, análisis de casos clínicos, seminarios o rondas clínicas, entre otras $(3,4)$.

El ejercicio médico exige resolver situaciones que comprometen la salud y la vida de los pacientes, por lo que es necesario que los médicos en formación desarrollen las conductas y habilidades necesarias para la toma de decisiones, ya sea en consulta externa, salas de hospitalización o de urgencias. En el caso del ECG, se requiere la integración de conocimientos en fisiología cardiovascular, semiología (signos y síntomas), componentes técnicos como la ubicación de los electrodos y calibración del equipo, al igual que una correcta lectura, análisis e interpretación de dicho examen.

\section{Los modelos pedagógicos y los cambios en la enseñanza}

En la enseñanza de la medicina ha predominado el modelo pedagógico tradicional, con bases paternalistas y el rol pasivo del alumno, donde el docente transmite el conocimiento de modo vertical y jerárquico asistido por la clase magistral. La evaluación, en consecuencia, es un recurso para verificar el aprendizaje, usualmente mediante pruebas escritas de tipo selección múltiple con única respuesta, método que estimula un aprendizaje repetitivo y memorístico. En este proceso, aunque efectivo, es común que el docente no logre percibir si el estudiante ha adquirido las capacidades suficientes para la comprensión e interpretación de pruebas diagnósticas, como el ECG (5).

Las nuevas tendencias pedagógicas, por su parte, dan protagonismo al estudiante, favoreciendo un aprendizaje significativo en los currículos de medicina y en las áreas de la salud. Muchos docentes se acercan empírica e intuitivamente a estos modelos al descubrir mejores resultados en las pruebas escritas, lo que podría traducirse en una mayor apropiación de los conceptos enseñados.

Se define el aprendizaje significativo como la adquisición de nueva información la cual se relaciona de manera no arbitraria (no literal, no memorística) con saberes que el estudiante ya conoce, estableciendo relaciones que construyen un nuevo saber con mayor sentido y significado (6). Así, en los primeros semestres de su formación, el estudiante adquiere una serie de conocimientos básicos que, al enlazarlos con conceptos ya aprendidos mediante relaciones lógicas y experienciales o emocionales, habrán de dar pie a la construcción de nuevos aprendizajes con mayor grado de complejidad, para el caso del presente estudio es lo que sucede con las prácticas de electrocardiografía y las tutorías por pares.

\section{Aprendizaje por pares: una estrategia didáctica activa}

Keith Topping define el aprendizaje por pares como: "Personas de grupos sociales similares, que no son profesores profesionales, ayudándose unos a otros a aprender y aprender ellos mismos mediante la enseñanza" (7); en tanto Ross y Cameron plantean como característica fundamental de la enseñanza por pares el que los tutores no son expertos en la disciplina ni en la enseñanza (8).

En el aprendizaje por pares el estudiante percibe estar hablando con un igual, en una relación horizontal que desplaza aquellas relaciones verticales o jerárquicas propias de la clase magistral. Bajo esta estrategia la figura del tutor par pasa a ser la del guía o mediador, donde el estudiante puede expresar sin temor sus ideas y concepciones. La dinámica de este escenario obliga a que el estudiante sea reflexivo acerca de su propio proceso de aprendizaje, haga uso de todos sus conocimientos y los ponga en juego tanto para el desarrollo de la práctica como para la discusión y el debate con los compañeros, fomentando el desarrollo de habilidades cognitivas como identificar y correlacionar.

\section{Cómo desarrollar una enseñanza por pares}

La aplicación de esta estrategia de enseñanza-aprendizaje en un curso de fisiología cardiaca se hizo mediante prácticas guiadas por tutores pares, con la toma, lectura e identificación de trazos electrocardiográficos entre compañeros y empleados (pacientes normales). Los objetivos del curso establecen, entre otros, la toma del electrocardiograma, calibración del equipo de electrocardiografía, poner los electrodos, identificar variables externas que alteren el registro, identificar la morfología normal del trazo electrocardiográfico y realizar la lectura e interpretación del electrocardiograma.

En el curso de cardiorrespiratorio, del tercer semestre del ciclo básico de formación, se abordan contenidos específicos de morfología, fisiología y electrocardiografía, previos a la tutoría por pares, actividad que se lleva a cabo por fuera de la programación regular del curso con un tutor par que acompaña a un grupo de seis alumnos y cuya asistencia es voluntaria y no conduce a una calificación. Los tutores pares son estudiantes de semestres avanzados quienes, además de tener cualidades como actitud y aptitud para enseñar, deben haber aprobado entre 40 y $90 \%$ de los cursos del pregrado y un promedio académico acumulado superior a 3.8. Los tutores novatos reciben capacitación por parte de el coordinador del curso y tutores avanzados (aquellos con más de dos semestres desarrollando las tutorías) quienes los acompañan en las primeras sesiones, les explican la dinámica y los entrenan en la metodología que se lleva a cabo en la actividad.

\section{Observaciones y discusión de las sesiones}

En el aprendizaje desarrollado por tutores pares se rompe con las barreras propias del modelo pedagógico tradicional, que usualmente intimidan y bloquean al alumno, y se establece una relación de intercambio de saberes que estimula una participación más activa de los alumnos, fundamental para precisar y aclarar los conceptos, principios y objetivos de la sesión (9). Al respecto, uno de los entrevistados manifestó:

No eran docentes, sino compañeros de la carrera, y si dominaban muy bien el tema, me parece que además de 
dominarlo tenían como esa vocación de docencia porque nos sabían explicar; porque uno puede tener mucho conocimiento pero no saber transmitirlo. (E01 - pl)

De acuerdo con Carolien Bulte et al (10), la congruencia cognitiva y social entre pares promueve una enseñanza adecuada en un ambiente tranquilo y seguro. El par reconoce las fortalezas y debilidades y hace énfasis en esos temas específicos para el alumno. En relación con este concepto uno de los estudiantes describe:

La tutoría de electrocardiograma nos la dieron unos estudiantes que están en un semestre superior, y fue muy bueno porque como ellos también son estudiantes que acaban relativamente hace poco de ver lo mismo, entonces saben cuáles son las debilidades y las fortalezas de los estudiantes, entonces saben cómo hacer énfasis en lo que deben reforzar que nos da más dificultad [...] (E02-p1)

Por su parte Bennett et al (11) consideran que la proximidad social entre pares posibilita que expresen más cómodamente las dificultades, se sientan más relajados y les genera confianza el que un compañero asuma el rol de docente y puedan expresar sus dudas, como lo manifiesta uno de los participantes de las tutorías:

Me pareció muy entendible la manera en que los tutores me explicaron cómo era la parte, sobre todo fisiológica, porque en la clase no había entendido. Lo de los vectores y todo eso no lo entendí; en cambio, ellos me lo explicaron de una manera muy amena. (E03 - pl)

En las tutorías el estudiante desarrolla habilidades prácticas, como la ubicación de electrodos y dirigir las condiciones que debe tener el paciente (estudiante simulado) para realizar el registro del ECG. Igualmente, identifica las variables que puedan alterar la toma de la prueba diagnóstica, seguido de un análisis e interpretación de los resultados. En estas prácticas se establece el escenario para lograr un aprendizaje significativo, dado el esfuerzo que hace el estudiante para establecer conexiones e interacciones con lo que está viviendo, lo cual lo induce a obtener pensamientos ordenados y adquirir una lógica con valor instrumental y operativo que le permita familiarizarse con futuros escenarios (12). Uno de los participantes relata:

[...] tuve la oportunidad de conocer cómo se ponían los electrodos y todo, lo que me pareció muy interesante y me permitió entender aún más qué era el electrocardiograma, todo lo referente a ello [...] (E04 - pl)

\section{Conclusiones}

El aprendizaje por pares se desarrolla con estudiantes de pregrado, y ello propicia un ambiente con relaciones horizontales lo que genera compromiso por el aprendizaje y mayor participación. La interacción entre el tutor par y los estudiantes facilita la comprensión de conceptos, identificar errores aprendidos y corregirlos oportunamente para evitar que se fijen en la memoria. Ambos, tutores y estudiantes, aprenden bajo una experiencia desarrollada por ellos mismos fortaleciendo la autonomía, las capacidades de autoaprendizaje y el trabajo en equipo(13).

Bajo esta estrategia didáctica, que favorece el aprendizaje significativo y la adquisición de destrezas y habilidades en los primeros años de formación, se observa el afianzamiento de los conocimientos con respecto a la lectura e interpretación del electrocardiograma y un mejor desempeño del estudiante en las pruebas escritas (datos no publicados en este estudio); además, el trabajar con grupos pequeños de estudiantes posibilita un acompañamiento más cercano en el proceso enseñanza-aprendizaje, apoyado por estudiantes de niveles superiores con un interés particular en la docencia.

\section{Agradecimientos}

Al Departamento de Fisiología y Bioquímica. Grupo de investigación EDUSALUD, Estudiantes tutores pares y la Facultad de Medicina.

\section{Referencias}

1. Coll, Y., Francisco, M., Valladares, D. J., Claudio, C., Rodríguez, M. G., Falcón, A., Habib, G. Infarto agudo de miocardio. Actualización de la Guía de Práctica Clínica Acute Myocardial Infarction. An Update of the Clinical Practice Guideline. Revista Finlay, 2016: 6(2), 170-190.

2. Roth, G. A., Johnson, C., Abajobir, A., Abd-Allah, F., Abera, S. F., Abyu, G., Murray, C. Global, Regional, and National Burden of Cardiovascular Diseases for 10 Causes, 1990 to 2015. Journal of the American College of Cardiology, 2017: 70(1), 1-25. Disponible en: https://doi.org/10.1016/j.jacc.2017.04.052

3. Fent, G., Gosai, J., \& Purva, M. Teaching the interpretation of electrocardiograms: Which method is best. Journal of Electrocardiology, 2015: 48(2), 190-193. https://doi.org/10.1016/j.jelectrocard. 2014.12.014

4. Raupach, T., Harendza, S., Anders, S., Schuelper, N., \& Brown, J. How can we improve teaching of ECG interpretation skills? Findings from a prospective randomised trial. Journal of Electrocardiology, (2016): 49(1), 7-12. Disponible en: https://doi.org/10.1016/j.jelectrocard.2015.10.004

5. Paulo Freire. (Pedagogía de la autonomía: saberes necesarios para la práctica educativa 2006. (S. XXI, Ed.2006). Pag 23-87.

6. Moreira,M.A.¿Al final, qué es el aprendizaje significativo? Revista Qurriculum (2012).

7. Topping, K. J. The effectiveness of peer tutoring in further and higher education: A typology and review of the literature. Higher Education, 2009, 32(3), 321-345. Disponible en: https://doi.org/10.1007/BF00138870

8. Ross, M. T., \& Cameron, H. S. (2007). Peer assisted learning: A planning and implementation framework: AMEE Guide no. 30. In Medical Teacher (Vol. 29).

9. Sammaraiee, Y., Mistry, R. D., Lim, J., Wittner, L., Deepak, S., \& Lim, G Peer-assisted learning: Filling the gaps in basic science education for preclinical medical students. Advances in Physiology Education, 2016 40(3), 297-303. Disponible en: https://doi.org/10.1080/01421590701665886

10. Bulte, C., Betts, A., Garner, K., \& Durning, S. (2007). Student teaching: Views of student near-peer teachers and learners. Medical Teacher, 29(6), 583-590. Disponible en: https://doi.org/10.1080/01421590701583824

11. Bennett, D., O'Flynn, S., \& Kelly, M. (2015). Peer assisted learning in the clinical setting: an activity systems analysis. Advances in Health Sciences Education, 20(3), 595-610. Disponible en: https://doi.org/10.1007/s10459-014-9557-x

12.Pawlina, W., \& Drake, R.L. (2016, January 1). Authentic learning in anatomy: A primer on pragmatism. Anatomical Sciences Education, Vol.9, pp. 5-7. Disponible en: https://doi.org/10.1002/ase.1592

13. Goodman, B. E., Barker, M. K., \& Cooke, J. E. (2018). Best practices in active and student-centered learning in physiology classes. Advances in Physiology Education, 42(3), 417-423. Disponible en: https://doi.org/10.1152/advan.00064.2018 